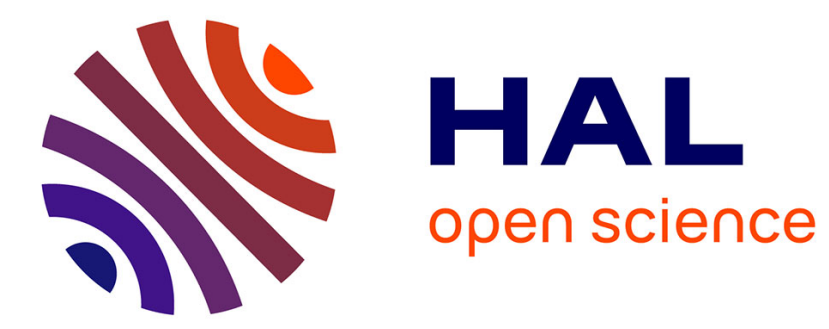

\title{
Le cut-up : ut pictura poesis au pied de la lettre Clémentine Hougue
}

\section{To cite this version:}

Clémentine Hougue. Le cut-up: ut pictura poesis au pied de la lettre. Trans : Revue de Littérature

Générale et Comparée, 2006, Littérature et image, 2, 10.4000/trans.159 . hal-02443727

\section{HAL Id: hal-02443727 \\ https://hal.science/hal-02443727}

Submitted on 17 Jan 2020

HAL is a multi-disciplinary open access archive for the deposit and dissemination of scientific research documents, whether they are published or not. The documents may come from teaching and research institutions in France or abroad, or from public or private research centers.
L'archive ouverte pluridisciplinaire $\mathbf{H A L}$, est destinée au dépôt et à la diffusion de documents scientifiques de niveau recherche, publiés ou non, émanant des établissements d'enseignement et de recherche français ou étrangers, des laboratoires publics ou privés. 


\section{TRANS-}

Revue de littérature générale et comparée

\section{Le cut-up : ut pictura poesis au pied de la lettre}

\section{Clémentine Hougue}

\section{OpenEdition}

Journals

Édition électronique

URL : http://journals.openedition.org/trans/159

DOI : 10.4000/trans.159

ISSN : 1778-3887

\section{Éditeur}

Presses Sorbonne Nouvelle

Ce document vous est offert par Le Mans Université

\section{T) \\ Université:Maine}

Référence électronique

Clémentine Hougue, "Le cut-up : ut pictura poesis au pied de la lettre », TRANS- [En ligne], 2 | 2006, mis en ligne le 22 juin 2006, consulté le 17 janvier 2020. URL : http://journals.openedition.org/trans/159 ; DOI : 10.4000/trans. 159

Ce document a été généré automatiquement le 17 janvier 2020

Tous droits réservés 


\title{
Le cut-up : ut pictura poesis au pied de la lettre
}

\author{
Clémentine Hougue
}

L'écriture a cinquante ans de retard sur la peinture. Je me propose d'appliquer les techniques des peintres à l'écriture ; des choses aussi simples et immédiates que le collage et le montage. (Brion Gysin, « Les cuts-ups s'expliquent d'euxmême ",

Euvre croisée, 1976 :39)

C'est en 1958 que William Seward Burroughs (1914-1997) invente, en collaboration avec le peintre et poète Brion Gysin (1916-1986), une technique d'écriture fondée sur le découpage et l'assemblage de textes : le cut-up. Naîtront de cette découverte trois romans qui se démarquent de façon significative des précédents textes de Burroughs, comme Junkie ou Le Festin Nu : La machine molle (1960), Le ticket qui explosa (1962) et Nova Express (1964). Cette trilogie entre en relation avec CEuvre croisée, un recueil de travaux de Burroughs et Gysin écrits entre 1965 et 1970, qui pose les principaux enjeux du cut-up : cette œuvre mêlant essais sur la poésie et textes de fictions, acquiert par sa composition un statut hybride entre texte et méta-texte, à la fois création littéraire et discours sur cette création. Le cut-up consiste en un découpage de différents textes, réassemblés dans le but de former un texte inédit, à la forme complexe, fragmentée : la déstructuration de la syntaxe et le morcellement de l'unité textuelle donnent à lire une prose haletante, dont le point de vue "ressemble à l'œil blessé d'une mouche" (Gérard-Georges Lemaire, «Préface», Trilogie, 1994: 21). Le cut-up pose comme condition préalable de mise en œuvre un décloisonnement entre littérature et art pictural: en effet, c'est sous l'impulsion des collages picturaux et des photomontages de Gysin que Burroughs aurait eu l'idée de procéder de façon similaire avec du texte. Le texte, en étant découpé, plié, collé, est traité comme une image, pris dans sa dimension sensible, matérielle. On serait alors en présence d'une sorte de remotivation postmoderne de la formule d'Horace, ut pictura poesis : il s'agit ici non plus d'établir une comparaison entre peinture et poésie 
quant au mode de représentation du réel, la mimesis, mais de poser entre ces deux arts une relation d'identité essentielle.

Le cut-up pose donc d'emblée la question du rapport entre l'image et le texte, d'abord du fait que cette technique d'écriture s'est accompagnée de travaux picturaux et cinématographiques, mais aussi parce qu'elle met au jour le problème de la mécanisation de l'écriture dans le découpage et le collage. Le décloisonnement de l'image sensible et du langage conceptuel rapproche le cut-up des interrogations philosophiques de Jacques Derrida et Gilles Deleuze concernant les rapports existant entre langage, écriture et sens. Le point de départ de cette zone d'affinité est une pensée de la multiplicité, contrecarrant une conception de l'écriture régie par des oppositions binaires: parole et écriture chez Derrida ; partie et tout chez Deleuze.

\section{Photomontage, cinéma, écriture}

3 On ne peut interroger le cut-up indépendamment des œuvres picturales et cinématographiques de Burroughs et Gysin, au risque d'éluder une part importante de leur démarche esthétique. Les trois domaines dans lesquels se déploie cette technique non seulement sont intimement liés mais procèdent d'un même mouvement de décomposition-recomposition. Le cut-up textuel trouve sa source dans l'art pictural. Ce sont en effet les collages de Brion Gysin qui influencèrent l'écriture de la trilogie $L a$ machine molle (1960), Le ticket qui explosa (1962), Nova Express (1964). Consécutivement à la rédaction de ses trois ouvrages, Burroughs réalise plusieurs films fondés sur la technique du cut-up, comme Towers open fire (1965) et The Cut-ups (1966). La multiplication des supports invite à placer le cut-up dans une logique de l'interpénétration des techniques, qui tend à annuler l'antagonisme de l'écrit (conceptuel) et de l'image (visuelle, sensorielle).

4 Si Brion Gysin est reconnu comme plasticien, on ignore encore largement les œuvres picturales de William Burroughs. Celles-ci s'avèrent pourtant fort éclairantes quant à sa vision de la littérature, puisqu'il s'agit en grande partie de collages, réunis dans le catalogue Ports of Entry, réalisé pour l'exposition des toiles et des scrapbooks (littéralement, carnets de fragments). Le collage en art plastique revêt deux particularités. D'une part, il est un agencement d'éléments et matériaux disparates, de formes, volumes, tailles et textures diverses. D'autre part, les éléments qui composent un collage sont, pour la plupart, des parties de l'objet préexistant dont ils ont été extraits (un morceau de journal déchiré, par exemple) : ainsi la partie de l'objet insérée dans le collage suggère le tout auquel elle appartenait originairement, mais devient dans ce processus la partie d'un autre tout: l'œuvre d'art. Dans le cut-up écrit, on retrouve une démarche similaire : les fragments de phrases sont issus de textes préexistants et recomposent un nouveau texte. Ce morcellement en propositions donne à lire un texte à la fois discontinu et formant un tout. De plus, les fragments qui composent les cut-ups sont souvent utilisés plusieurs fois : ainsi, une proposition ou un syntagme apparaitra à divers endroits du texte, créant alors une connexion entre deux parties du roman sans autre lien apparent. Le texte se développe d'une part dans le mouvement des éléments extérieurs insérés dans le collage, d'autre part dans un mouvement interne de ces éléments qui surgissent ici ou là dans l'œuvre. Ainsi les fragments sont-ils toujours en mouvement : mouvement du dehors $\mathrm{du}$ texte vers son dedans, puis à l'intérieur même du texte. Enfin, les œuvres cinématographiques précédemment citées donnent à voir des séquences se succédant 
sans lien logique : c'est le montage seul qui lie ces plans entre eux, et non la signification des scènes. Là encore, des séquences apparaissent plusieurs fois. Les collages picturaux, les cut-ups ou les œuvres filmiques de Burroughs et Gysin partagent les mêmes points d'ancrage: l'hétérogénéité, la discontinuité, la répétition. Il s'agit là de concepts fondamentalement liés à la modernité et plus particulièrement à la reproductibilité des matériaux qui composent les œuvres. "À la plus parfaite reproduction manquera toujours quelque chose : le hic et nunc de l'œuvre d'art - l'unicité de son existence au lieu où elle se trouve » (Walter Benjamin, $2000: 273$ ) : la perte de l'unicité dont parle Benjamin s'applique dans le cut-up; la reproduction des segments, leur répétition, produit une dislocation du discours qui ouvre la voie à une prolifération multidirectionnelle et simultanée du sens, une structure en réseau, ou encore, en termes deleuziens, à un rhizome. Cette matérialité de l'écrit à l'œuvre dans les textes de William Burroughs et Brion Gysin, ainsi que l'interchangeabilité des propositions revient alors à une spatialisation du langage dans l'espace même du livre. La répétition des fragments opère comme une variation chromatique du sens, qui varie en fonction des segments précédents et suivants, comme des sérigraphies éclatées et disséminées dans le texte. Cuvre croisée présente bien les relations d'interpénétrations entre image et texte, en mêlant photomontage, synopsis de films, cut-ups textuels et réflexion théorique. En compilant cinq années de travail des deux auteurs, cet ouvrage trouve sa cohérence dans une réflexion transdisciplinaire sur la mécanisation de la production du sens.

\section{Écriture organique, écriture mécanique}

5 To cut-up signifie découper: il s'agit d'un geste mécanique, qui diffère de l'acte de déchirer, puisqu'il requiert l'intervention d'un outil. Le cut-up serait alors une écriture mécanique. Jacques Derrida, dans Papier Machine, explique que la machine est sans organicité : elle est répétition, sans désir ni intention, programme calculable n'ayant besoin de personne. Au contraire, l'événement est une singularité non programmable, c'est une expérience subie par quelqu'un, advenant de manière sensible ou « aesthésique ». En ce sens, l'événement est essentiellement organique, «parce qu'il n'est pas de pensée de l'événement, semble-t-il, sans une sensibilité, sans un affect esthétique et quelque présomption d'organicité vivante " (Jacques Derrida, "Le ruban de machine à écrire ", 2001 : 35). Dans la logique traditionnelle, le mécanique et l'organique s'excluent. L'écriture littéraire comme création de fiction, est de l'ordre de l'organique, puisqu'elle est une expérience singulière, nécessitant un auteur et un lecteur : du point de vue de sa mise en œuvre comme de celui de sa réception, le texte est un événement singulier. Une forme littéraire faisant intervenir une mechanê ( $\mu \eta \chi \alpha v \eta)$ mettrait au jour une nouvelle logique qui serait à la fois un événement organique et une mécanique inorganique : le cut-up procède de cette logique.

6 Le titre original d'Euvre croisée (The third mind) nous met sur la piste de cet événement inouï, à la fois mécanique et organique : l'émergence de ce que nous appellerons une « troisième voix ». En découpant et réagençant plusieurs textes, on observe l'apparition d'un sens, d'une voix, d'un « esprit » (mind), qui n'est ni celui d'un texte ni celui de l'autre. Cette «troisième voix » est ainsi celle qui, née du hasard, ne peut se manifester, se faire entendre, que dans le collage de deux textes: un tel constat met en question la notion même d'auteur. Le cut-up, comme nous l'expliquions en préambule, consiste en un agencement aléatoire de différentes parties d'un texte préalablement découpé : ainsi, le 
cut-up n'existe pas avant sa conception, c'est-à-dire qu'il n'est pas, comme un poème ou un roman, pensé ou imaginé au préalable, mais bien le fruit d'une composition hasardeuse d'où émerge un sens qui ne peut être préétabli avant le moment du montage. Dans le cut-up, il n'y a rien avant l'acte de cet agencement. Le texte manifeste une réelle indépendance vis-à-vis de son auteur: c'est bien la mécanisation de l'écriture, de la création a priori essentiellement organique, qui fait l'œuvre, qui fait qu'il y a une œuvre, que quelque chose arrive, donc qu'il y a évènement.

7 Par ailleurs, la performativité du cut-up participe également de cette mise en relation du mécanique et de l'organique. Comme un automate, une mécanique autonome, le cut-up parle de lui-même : il est à la fois présentation d'une fiction et présentation des conditions de sa propre réalisation. Le texte de Brion Gysin intitulé « Les cut-ups s'expliquent d'euxmêmes ", dans Euvre croisée (1976 : 39-46), est particulièrement significatif à ce sujet : il se compose d'un texte expliquant les tenants et les aboutissants du cut-up, puis un découpage de ce même texte en trois colonnes et une série de différents réagencements selon diverses combinaisons possibles. Il s'agit donc à la fois d'un jeu sur le langage, d'une mise en scène de l'écriture et d'un art poétique, dans le sens où il est à la fois poésie et explication de cette poésie, texte et métatexte. Dans le cadre de ce texte, qui est à la fois présentation de lui-même et présentation des conditions de sa mise en œuvre, la dimension performative est poussée à l'extrême : l'œuvre se fait à mesure qu'elle dit se faire. Mais plus globalement, toute l'entreprise fictionnelle de cette trilogie repose sur cette ambivalence : le cut-up est à la fois intérieur (en tant qu'œuvre) et extérieur à luimême (en tant que discours sur lui-même). Dans ce jeu réflexif, l'œuvre est à la fois même et autre, identité et répétition, organique et mécanique. Elle présente les conditions de sa production en même temps qu'elle se présente comme œuvre. Le cut-up semble donc faire le lien entre des principes antagonistes, ce qui induit de nombreuses questions quant à sa portée conceptuelle concernant le langage, l'écriture et le sens.

\section{Implications conceptuelles}

8 Cette méthode d'écriture procède d'un questionnement sur le langage, comme le point de départ qui motive sa mise en œuvre. Il s'agit pour Burroughs de mettre en question le rapport du langage au texte et du texte au sens; le cut-up serait alors un « formidable mouvement d'inquiétude sur le langage» (Jacques Derrida, 1967b : 9). L'inquiétude, du latin inquies, désigne communément un état d'agitation, une absence de repos. Le terme quies, dont il est une composition, signifie "repos", "sommeil», mais une autre acception du terme veut également dire «silence » ou « inaction » : l'inquies est donc un mouvement de rupture du silence, d'entrée en action, le passage d'un état passif à un état actif. Agir, produire un discours, mettre en mouvement la matière même de son texte : la définition de Deleuze et Guattari de la philosophie comme «l'art de former, d'inventer, de fabriquer des concepts » (Gilles Deleuze et Félix Guatarri, 1991: 8) traduit bien l'idée de cette mise en mouvement, de ce passage à l'action, qui nous invite à envisager le cut-up comme réflexion philosophique sur le lien entre langage et écriture.

Comme mécanisation de l'organicité, ces textes se trouvent à la croisée des chemins de deux concepts de la philosophie contemporaine française : la déconstruction de Jacques Derrida et la notion de rhizome de Gilles Deleuze. Le cut-up est révélateur de la pensée contemporaine de la multiplicité et de la non-contradiction de couples de notions antagonistes (notamment écriture/parole, ou comme nous venons de le voir, mécanique/ 
organique), que l'art et la philosophie vont interroger durant la deuxième moitié du $\mathrm{XX}^{\mathrm{e}}$ siècle. Aussi les recherches de Burroughs et Gysin procèdent-elles de cette réflexion sur l'écriture comme déploiement du sens non linéaire et non hiérarchique, c'est-à-dire d'une part ne subordonnant plus l'écriture à la parole et d'autre part ne soumettant plus le sens à l'organisation du langage.

\section{Une poétique de la déconstruction}

Nous l'avons vu, ces textes exposent un projet en même temps qu'elles le mettent en œuvre : faire une écriture mécanique, indépendante de son auteur, en somme une pure présentation sans sujet individuel. Quel est alors ce langage autogénéré ? Un langage dont l'origine ne serait plus la parole, mais l'écriture, c'est-à-dire un langage qui viendrait au jour dans sa manifestation sensible, et non plus dans l'abstraction d'une parole première? Les éléments de réponse se trouvent sans doute dans le questionnement récurrent sur le Verbe, que l'on trouve par exemple dans Le Ticket qui explosa (« Vous rencontrerez un organisme résistant qui vous force à parler. Cet Organisme c'est le verbe. $\mathrm{Au}$ commencement était le verbe. Au commencement de quoi exactement?» [Trilogie, 1994 : 206]), mais aussi dans le texte de Brion Gysin, « Minutes to go I » : ce pourrait bien être le dernier mot partout / ce n'est pas le commencement au commencement était le verbe / le verbe a existé depuis trop longtemps / vous dans le verbe le verbe dans vous / nous sommes dehors / vous êtes dedans. » (Euvre croisée, 1976 : 48.)

11 Le "verbe du commencement» est un motif qui hante littéralement les cut-ups de Burroughs et Gysin : il s'agit là de mettre en question l'existence d'un signifié originel, d'une parole précédant le langage, qui serait l'essence du langage. Une telle démarche entre en résonance avec les travaux de Jacques Derrida qui, dans De la grammatologie, nomme cette "métaphysique du langage» le logocentrisme (1967a:11). Selon ce philosophe, le concept occidental de langage «confine l'écriture dans une fonction seconde et instrumentale, traductrice d'une parole pleine et pleinement présente (présence à soi, à son signifié, à l'autre...) » [Ibid. :17]. Il montre ainsi que la dévaluation de l'écriture traverse toute la pensée métaphysique de Platon à Heidegger, et que l'art d'écrire est toujours posé comme "destruction croissante du mot ou de la parole" (Jacques Derrida, 1990: 202). Il s'agit donc de repenser l'écriture, et avec elle toute la littérature, hors de cette détermination métaphysique. On trouve dans le cut-up l'ébauche de ce remaniement. Le mot y est considéré pour lui-même, et la spatialisation du texte dont il était question plus haut participe de ce processus. En effet, cette technique présente une revalorisation du signifiant, non plus simple moyen dans le langage, coquille creuse qui abrite le signifié, mais totalité indépendante de toute détermination qui lui serait extérieure. Le mot pris dans sa matérialité se déploie en tant que lui-même, est présent en tant que lui-même. Dans le cut-up, le jeu visuel d'agencement du texte en fragments donne son sens au signifiant. En ce sens, l'œuvre se manifeste dans l'apparitiondu mot, de la proposition, et non sous l'autorité d'une origine qui de tout temps lui préexisterait. D'un signifiant originel qui serait pure présence, on passe à la présentation de la trace, de ce «supplément » dont parle Rousseau et que glose Derrida dans De la grammatologie. Le cut-up est une réfutation de la métaphysique du langage. C'est bien l'écriture en tant que telle qui fait sens, et non plus le spectre d'un signifié qu'elle ne ferait que "signaler», comme l'explique Derrida dans L'écriture et la différence: 
C'est quand l'écrit est défunt comme signe-signal qu'il naît comme langage ; alors il dit ce qui est, par là même ne renvoyant qu'à soi, signe sans signification, jeu ou pur fonctionnement, car il cesse d'être utilisé comme information naturelle, biologique ou technique, comme passage d'un étant à l'autre ou d'un signifiant à un signifié.

(1967b : 23)

12 Les affinités de la déconstruction et de la technique à l'œuvre dans les travaux de Burroughs et Gysin ne sont pas simplement celles qu'entretiennent une pensée philosophique et une pratique artistique. Nous l'avons déjà montré : le cut-up, ainsi que les collages et les films, coïncident avec une importante réflexion théorique de Burroughs et Gysin. Et inversement, Jacques Derrida a mis en texte cette déconstruction, dans Glas (1974) ou dans la première partie de La carte postale (1980). En outre, les recherches sur le cut-up montrent également d'importants points de convergence avec la philosophie de Gilles Deleuze, qui écrivait avec Félix Guattari : «Le contenu n'est pas un signifié, ni l'expression un signifiant, mais tous deux sont les variables de l'agencement.» (1980:115).

\section{Le cut-up comme rhizome}

Dans l'écriture du cut-up, le texte se libère de la linéarité et de la hiérarchie qu'impose le langage à la construction du sens. Plus de narration, de déroulement ou de chronologie : le texte est envisagé comme un ensemble d'unités à la fois autonomes et interdépendantes, comme une surface mouvante sans architecture ni organisation, un " corps sans organes ». Ainsi, "les poèmes permutés font tourner les mots tout seuls; partant en échos quand se permutent les mots d'une phrase puissante pour faire des ondulations toujours en expansion de sens dont ils ne semblent pas capables quand on les a frappés et ensuite collés dans cette phrase » (Brion Gysin, «Les cuts-ups s'expliquent d'eux-mêmes », Euvre croisée, 1976 : 39-40). C'est la construction de la langue qui permet la venue au jour du sens : par exemple, la structure syntaxique détermine les liens de causalité entre les propositions, liens qui vont relier deux évènements du monde ensemble. En rompant avec la syntaxe, les propositions ne sont plus connectables entre elles suivant cette logique : le sens emprunte donc une autre voie. Prenons pour exemple un extrait du Ticket qui explosa : « Flashes incontrôlés explosèrent - Une bonne sensation Le langage des virus (ce que sont ces expériences) vraiment nécessaires? Message de la vie écrit "Nous sommes venus pour manger" ?? -.» (Trilogie, 1994: 245). L'absence de liens logiques entre les propositions situe ce texte entre sens et non-sens, ce qui s'apparente à la définition du paradoxe que livre Gilles Deleuze dans Logique $d u$ sens : « Le paradoxe est d'abord ce qui détruit le bon sens comme sens unique, mais ensuite ce qui détruit le sens commun comme assignations d'identités fixes.» (1969:12). Dans le processus de découpage, l'identité est toujours renouvelée, elle perd sa fixité jusqu'à se perdre comme identité. "Une page de Rimbaud découpée et réarrangée donnera des images tout à fait différentes. Des images de Rimbaud, vraiment de Rimbaud, mais nouvelles ", affirme Burroughs (Entretiens, 1966, notre traduction). L'identité, dans ce processus de répétition par truchement du montage, devient autre en restant la même, et crée une unité hétérogène. Dans les trois romans de Burroughs, récit de l'auteur et pensées des personnages sont ainsi emportés dans un même flot d'images fugaces, instables, apparaissant sur un même plan narratif : les récits ne sont pas imbriqués les uns dans les autres, il n'y a pas de hiérarchie des discours, mais toutes les images forment une surface sans début ni fin. La partie est le tout et inversement, comme l'explique Vito 
Amoruso: "le livre, et encore moins le roman, en tant qu'unité empirique de la recherche, comme sa coagulation temporaire, n'existe plus et tend à se réfléchir dans la page elle-même, et à la limite, dans le mot seul par une sorte de processus de fixation nucléaire du moment visuel isolé. » (1975:139).

Dans cette configuration « rhizomique », le texte devient un corps sans organes. Dans un des textes fondateurs de sa pensée, « Rhizome » (1980: 9), Gilles Deleuze explique qu'« un rhizome est fait de plateaux» ( Ibid.: 32), et «chaque plateau peut-être lu à n'importe quelle place et mis en rapport avec n'importe quel autre » (Ibid. : 33). En effet,

un rhizome ne commence ni n'aboutit pas, il est toujours au milieu, entre les choses

[...] Entre les choses ne désigne pas une relation localisable qui va de l'une à l'autre

et réciproquement mais une direction perpendiculaire, un mouvement transversal

qui les emporte l'une et l'autre, ruisseau sans début ni fin, qui longe ses deux rives

et prend de la vitesse au milieu. (Ibid. : 36-37)

Ainsi dans l'écriture, « le dehors n'a pas d'image, ni de significations, ni de subjectivité. Le livre est un agencement avec le dehors, contre le livre-image du monde» (Ibid. : 34). De même dans La machine molle, Le ticket qui explosa et Nova Express, le texte est pour luimême, hors de tout contexte et de toute représentation: il n'est que sa propre image, reflétée en elle-même à l'infini. Il en va de même pour la division en chapitres: les sections, qui se succèdent sans véritable continuité dans la narration, forment ainsi plusieurs récits indépendants les uns des autres, mais entretenant tous des liens. Construits en "plateaux », ces romans présentent bien une structure en rhizome, dont toutes les parties sont à la fois autonomes et connectables les unes aux autres. Dans des contextes toujours différents, la répétition d'un fragment, presque invisible, fait cohésion entre les passages, sans que ce lien soit d'ordre logique ou narratif. Le texte fonctionne ici comme un «système acentré, non hiérarchique, non signifiant, sans général, sans mémoire organisatrice ou automate central, défini par une circulation d'états » (Ibid. : 32). Le récit ne se déroule pas en fonction d'une chronologie qui tiendrait compte de ce que le lecteur sait et de ce qu'il doit encore apprendre pour donner à la diégèse, pour reprendre la terminologie de Gérard Genette, une unité; le texte se déploie en diverses surfaces traversées de mouvements communs. Comme le remarque encore Vito Amoruso, chez Burroughs

la subdivision en chapitres n'a maintenant qu'une fonction résiduelle et minime pour ainsi dire uniquement typographique - de scansion approximative de la matière informe et rutilante [...]. La page tend toujours plus à dépasser le tout pour s'affirmer isolément et sans relation par rapport au contexte, à se scinder à l'intérieur d'elle-même en atomes et en très fine poussière, en éclairs d'images. (1975 : 139)

Les cut-ups sont bien des textes-rhizomes, des agencements sans articulations prédéfinies, ou le texte morcelé agit comme un corps sans organes.

17 À la fois processus de déconstruction et structure en rhizome, le cut-up dissout simultanément les conceptions traditionnelles de parole et de texte, et de ce fait les deux conditions de l'unité du sens et de son intégrité dans le langage. Le texte n'est pas image du sens, il est création d'images pour elles-mêmes, avènement d'un sens inédit que seul le processus de mécanisation peut mettre en œuvre. En choisissant de traiter texte et image dans un même mouvement, William Burroughs et Brion Gysin réinvestissent simultanément les deux champs de création: le texte acquiert une matérialité qui lui donne une dimension nouvelle, l'image perd la fixité à laquelle la bornait la notion de représentation. Un ut pictura poesis au pied de la lettre, une lettre en perpétuel 
mouvement, dont les circonvolutions bouleversent le langage jusqu'à l'abolition de son verbe fondateur, jusqu'à la mise en péril de l'unilatéralité du sens.

De telles expérimentations ne sont certes pas inédites (on pense naturellement aux découpages de Tristan Tzara, aux cadavres exquis des surréalistes ou à l'emploi de coupures de presse dans les romans de Dos Passos), mais sans doute nos auteurs se démarquent-ils radicalement de leurs prédécesseurs par le degré de systématisation atteint dans leur démarche esthétique. Au point extrême où, comme dans les plus inquiétants romans d'anticipation, cette machine à écrire devient autonome, comme le remarque Gérard-Georges Lemaire dans la préface d'Guvre Croisée: «La machine Burroughs, systématique et répétitive, déconnectante et reconnectante dans le même mouvement [...] échappe en dernier ressort au contrôle de son manipulateur, dans le sens où elle permet la mise en chantier des livres innombrables qui finissent par s'autoreproduire.» (1976: 15). En véritables spécialistes du scandale, Gysin et Burroughs s'attellent au démembrement de toutes les certitudes du lecteur en inventant une écriture de l'instable, toujours au bord de l'explosion.

\section{BIBLIOGRAPHIE}

Amoruso, Vito, 1975, «Le cut-up et la technique de la fugue », in Entretiens, Beat Generation, sous la direction de Yves Le Pellec, Rodez, Éditions Subervie, p. 133 à 141.

Benjamin, Walter, 2000,L'œuvre d'art à l'ère de sa reproductibilité technique, in CEuvres III, (dernière version 1939), trad. M. de Gandillac, Paris, « Folio », Gallimard.

Deleuze, Gilles, 1969, Logique du sens, Paris, coll. «Critique », Minuit.

Deleuze, Gilles, et Guattari, Félix, 1980, Capitalisme et Schizophrénie : Mille Plateaux, Paris, Minuit.

-, 1991, Qu'est-ce que la philosophie ?, Paris, Minuit.

Derrida, Jacques, 1967a, De la grammatologie, Paris, coll. « Critique », Minuit.

—, 1967b, L'écriture et la différence, Paris, « Tel quel », Seuil. Réédition 1979, « Points Essais, Seuil.

,- 1974, Glas, Paris, Galilée.

-, 1980, La carte postale, de Socrate à Freud et au-delà, Paris, Aubier-Flammarion.

-, 1990, Heidegger et la question, Paris, « Champs », Flammarion.

-, 2001, Papier machine, Paris,Galilée.

Sobieszek, Robert A., 1996, Ports of entry, William S. Burroughs and the Arts, Los Angeles County Museum of Arts, Thames and Hudson.

\section{Fuvres de William S. Burroughs}

1976, Cuvre Croisée, avec Brion Gysin, préface Gérard-Georges Lemaire, traduction GérardGeorges Lemaire et Christine Taylor, Paris, Flammarion. 
1990, Lettres de Tanger à Allen Ginsberg, traduction, Paris, Christian Bourgois Éditeur.

1994, Trilogie : La machine molle, Le ticket qui explosa, Nova express, préface Gérard-Georges Lemaire, traduction Mary Beach et Claude Pélieu, Paris, Christian Bourgois Éditeur.

\section{Textes originaux}

1966, Entretien avec Conrad Knickerbocker, Paris Review.

1978, The Third Mind, New York, Viking.

1987, The Ticket that Exploded, New York, Grove Press.

1992, Nova Express, New York, Grove Press.

1992, The Soft Machine, New York, Grove Press.

\section{RÉSUMÉS}

Le cut-up, créé par Brion Gysin et William Burroughs au début des années 1960, pose le problème des relations entre écriture et image. Il s'agit d'un agencement aléatoire de fragments de textes, qui met ces deux arts sur un même plan: profondément lié aux arts picturaux (Burroughs a réalisé plusieurs collages dans des carnets et des peintures), le cut-up met en question les conditions de production du sens, en créant un art d'écrire mécanique et répétitif.

The cut-up, invented by Brion Gysin and William Burroughs in the early 1960s, tackles the problem of the relationships between writing and images. It consists of an aleatory combination of scraps of texts, which establishes a connection between the visual and the textual: very close to pictorial arts (Burroughs realized several scrapbooks and paintings), it questions the conditions of production of meaning, by creating a mechanical and repetitive art of writing.

El "Cut-up", creado por Brion Gysin y William Burroughs a principios de los años 60, plantea el problema de las relaciones entre la escritura y la imagen. Se trata de un ordenamiento aleatorio de fragmentos de textos, qui pone en el mismo plano ambas artes : con una gran influencia de las artes pictóricas (Burroughs realizo numerosos collages en cuadernos de apuntes y en pinturas), el cut-up cuestiona las condiciones de producción del sentido al crear un arte mecánico y repetitivo.

\section{AUTEUR}

\section{CLÉMENTINE HOUGUE}

Master 2 à l'UFR de littérature générale et comparée de Paris III, sous la direction de Mr Bessière. Mémoire de DEA : "Le cut-up et la déconstruction : introduction à une étude comparée" (rapprochement entre la pensée de Derrida, le logocentrisme, la trace, l'itérabilité constitutive du langage, et les expérimentations textuelles de Burroughs et Gysin) 